DOI: $10.3901 / J M E .2021 .06 .184$

\title{
锯齿错列胁片尾气换热器的热电性能优化*
}

\author{
何 为 姚光森 蔡紫涵 石颖 杨添会 王誉霖 \\ (天津商业大学天津市制冷技术重点实验室 天津 300134)
}

\begin{abstract}
摘要: 由于尾气传热性能差, 尾气余热热电转换系统中常采用具有强化换热结构的尾气换热器, 其中, 锯齿错列肋片式是常 见型式之一。但如何平衡传热性能和流动阻力实现对换热器内部结构和整体尺寸的优化, 使得热电系统获得总的净输出功率 最大, 是值得研究的内容。但现有成果均是基于限定尺寸结构, 对换热器局部尺度及热电性能进行分析, 没有针对强化换热 器的整体优化结构和最大净输出功率展开研究。建立基于汽车尾气余热回收的热电转换数值模型, 结合场协同理论, 分析锯 齿错列肋片式尾气换热器内部肋片结构对热电功率的影响。此外, 综合考虑传热性能与流动阻力的平衡, 以热电转换最大净 输出功率为优化目标, 对热电模块结构进行优化, 并获得热电系统的相应最优性能。研究结果表明, 固定热电模块尺寸时, 净输出功随肋片高度增加明显增加, 但受肋宽影响较小; 对热电模块尺寸进行优化时, 肋高和肋宽的变化均对热电模块的最 优尺寸产生较大影响, 单位面积最大净输出功随肋宽增加而明显降低, 但受胁高的影响较小。
\end{abstract}

关键词: 热电转换; 强化换热; 锯齿错列肋片; 尾气余热

中图分类号: TM617

\section{Optimization of Thermoelectric Performance of Offset-strip Fin Type Exhaust Heat Exchanger}

\author{
HE Wei YAO Guangsen CAI Zihan SHI Ying YANG Tianhui WANG Yulin
}

(Key Laboratory of Refrigeration Technology of Tianjin, Tianjin University of Commerce, Tianjin 300134)

\begin{abstract}
Due to the poor heat transfer performance of exhaust gas, exhaust heat exchangers with enhanced heat exchange structure are often used in exhaust waste heat thermoelectric conversion systems. Among them, the offset-strip fin type is one of the common types. However, how to balance heat transfer performance and flow resistance to optimize the internal structure and overall size of the heat exchanger, so that the thermoelectric system can obtain the maximum total net output power, is worthy of research. However, the existing results are based on the analysis of the local size and thermoelectric performance of the heat exchanger based on a certain limited size structure, and there is no research on the overall optimized structure and maximum net output power of the enhanced heat exchanger. A thermoelectric conversion numerical model based on automobile exhaust heat recovery is established, combined with field synergy principle, and the influence of the internal fin structure of the offset-strip fin type exhaust heat exchanger on the thermoelectric power is analyzed. In addition, comprehensively by considering the balance of heat transfer performance and flow resistance and taking the maximum net output power of thermoelectric conversion as the optimization goal, the thermoelectric module structure is optimized, and the corresponding optimal performance of the thermoelectric system is obtained. The research results show that the net output power increases significantly with the increase of the fin height, but is less affected by the width of the fin when the size of the thermoelectric module is fixed. However, when the size of the thermoelectric module is optimized, the changes of the fin height and the fin width have great impact on the optimal size of thermoelectric module. The maximum net output power of per unit area decreases significantly with the increase of fin width, but is less affected by fin height.
\end{abstract}

Key words: thermoelectric conversion; enhanced heat transfer; offset-strip fin type; exhaust waste heat

* 国家自然科学基金(51806152)和国家科技部国际合作(2017YFE0198000)资 助项目。20200809 收到初稿, 20201210 收到修改稿 


\section{0 前言}

近年来, 基于半导体热电材料的制冷 ${ }^{[1-2]}$ 及发电 技术 ${ }^{[3]}$ 研究愈加受到重视, 汽车尾气余热热电转换 技术成为国际研究热点。基于温差发电原理的汽车 尾气余热利用, 是一项绿色环保发电技术, 它通过 半导体热电材料直接将低品位热能转化为电能, 无 需化学反应或流体工质参与循环做功, 发电成本低, 工作可靠, 具有结构紧凑、免维护、无噪声等优 点 ${ }^{[2]}$ 。其工作原理在于热电材料的塞贝克效应, 将 $\mathrm{P}$ 型和 $\mathrm{N}$ 型两种不同类型的半导体材料一端相连形成 一个 PN 结, 置于高温热源环境, 另一端形成低温 冷端, 在热激发作用下, $(\mathrm{P}) \mathrm{N}$ 型材料高温端空穴(电 子)浓度高于冷端, 在浓度梯度驱动下, 空穴和电子 开始向冷端扩散, 从而形成电动势, 完成将高温输 入的热能直接转化成电能的过程。目前有研究表明 热电材料转换效率已达到 10\%(甚至可以达到 $15 \%$ ), 从汽车废热中可以回收的能量大约占到整个燃油燃 烧化学能的 7\%左右 ${ }^{[4]}$ 。虽然我国对此方面的研究起 步较晚, 但近年来随着研究日益加深, 某些方面的 科研水平已达到甚至领先国际同行 ${ }^{[5]}$ 。

提及热电转换技术, 固然离不开高性能的热电 材料, 但应用于汽车尾气余热回收时, 还存在着许 多典型的工程热物理难题函待解决 ${ }^{[6]}$ 。其中, 针对 高效能尾气换热器的研究就是其中一个重点, 考虑 到尾气侧流体传热性能较差, 采用强化传热的换热 器形式是必要的。目前, 众多学者针对尾气强化传 热换热理论已展开大量研究 ${ }^{[7-8]}$ : LESAGE 等 ${ }^{[9-12]}$ 在 换热通道内设置不同型式的金属条插入物、扰流板 或扰流管等, 研究其强化传热和流阻特性; SHI 等 ${ }^{[13]}$ 提出基于扩展面积的紧凑式换热结构理论;

ESARTE 等 ${ }^{[14]}$ 探索了不同加肋强化换热方式(螺旋 型、之字型、直肋型)的综合传热效果; BAKER 等 ${ }^{[15]}$ 对光滑单层平板式、三层平板并联流道式、三层平 板蛇形流道串联式、平直翅片式、以及全填充金属 泡沫式换热结构的换热和压降性能进行了比较分 析; LU 等 ${ }^{[16]}$ 研究了采用矩形偏置肋片式和管内全 填充铝/铜金属泡沫的传热性能, 并对泵功损耗评 估, 发现尾气流量小时全填充金属泡沫具有更好优 势, 而流量大时由于产生阻力较大, 其净输出功不 及肋片式; WANG 等 ${ }^{[17]}$ 试验研究了采用通道内全填 充铜金属泡沫的强化换热方式，结果表明比不填充 时的系统总转换效率有大幅提高。

相比光滑平板式结构, 采用强化换热的换热器
结构一方面会带来传热性能的提高, 另一方面也会 带来流动阻力的提高。如何平衡传热性能和流动阻 力实现对换热器内部结构和整体尺寸的优化, 使得 热电系统获得总的净输出功率最大, 是值得研究的 内容。但现有成果均是基于一定限定尺寸结构, 对 换热器局部尺度及热电性能进行的分析, 没有针对 强化换热器的整体优化结构和最大净输出功率展开 研究。本文将主要基于带锯齿错列肋片的尾气强化 换热器结构, 通过平衡传热性能与流动阻力, 探索 其换热器优化结构与高效热电转换性能。

\section{1 数学模型}

\section{1 热电模型}

温差发电系统数值模型见图 1, 采用顺流换热, 冷却水冷却方式。图 1a 显示热电系统总体结构图, 采用光滑矩型平板换热器通道。两个热电模块置于 废气换热器通道两侧, 负载电阻 $R_{L}$ 等于热电模块 总内电阻。一对 PN 结由铜连接片串连接在一起, 且置于两片陶瓷片之间。两个冷却器置于热电模块 的冷端。单热电模块的 PN 结布置如图 $1 \mathrm{~b}$ 所示。整 个热电模块沿流体流动方向 $\left(x\right.$ 方向)排列 $n_{x}$ 个 $\mathrm{PN}$ 对, 与之垂直方向 $\left(y\right.$ 方向)排列 $n_{y}$ 个 PN 对, 全部 $n_{x} \times n_{y}$ 个 PN 对单元串联组成一个整体热电模块。图 $1 \mathrm{c}$ 显示热电系统热阻分布图。当热量由热流体传至 热电模块热端表面, 共 5 部分热阻: 热流体对流换 热热阻 $R_{i}$, 废气换热器导热热阻 $R_{h 1}$; 废气换热器 和热电模块间的接触热阻 $R_{f}$ fcon; 陶瓷片导热热阻 $R_{h 2}$; 铜导电片导热热阻 $R_{h 3}$ 。与此类似, 当热量由 热电模块冷端放热至冷流体侧时, 相应同样存在五 部分热阻 $R_{c} 、 R_{L 1} 、 R_{c c o n} 、 R_{L 2} 、 R_{L 3}$ 。计算中, 由于 冷流体为水, 比热及对流传热系数都较废气换热器 侧大, 沿程温度变化小, 这里为方便不考虑冷却水 沿程温度变化对对流换热系数的影响, 即假定冷却 水具有定对流换热系数。

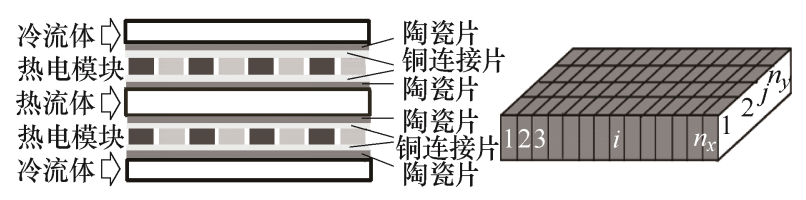

(a) 结构原理

(b) 单热电模块平面布置图

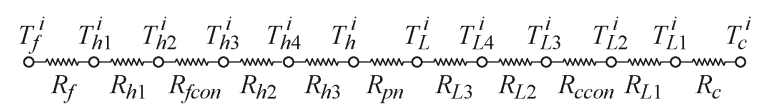

(c) 热阻分布

图 1 热电系统数学模型

模型假设： $\mathrm{P}$ 型和 $\mathrm{N}$ 型热电材料具有相同的尺 
寸和物性参数, 且热电性能稳定。热电偶臂间无空 气, 所有热辐射忽略, 忽略汤姆逊效应, 忽略换热 器横向导热。热电模块和换热器间接触热阻取 $R_{f c o n}$ $=R_{\text {ccon }}=0.0008 \mathrm{~m}^{2} \cdot \mathrm{K} / \mathrm{W}$ 。

对于每个热电模块, 由于所有 PN 热电单元串 联联接, 每个 PN 单元的电流等于整个热电模块总 电流。沿 $y$ 向, 所有小热电单元几乎具有相同的热 电温度, 因此, 假定 $y$ 向每一行热电单元为一个新 的小计算单元。因此, 以新计算单元为标识, 所有
变量以上角标 $i$ 来区别沿 $x$ 方向的小热电单元。对 于小热电单元 $i$, 热流体释放到热电模块热端的热量 为 $q_{h}{ }^{i}$, 热流体温度由 $T_{f}{ }^{i}$ 降为 $T_{f}{ }^{i+1}$, 与此同时热电 模块热端温度被加热到 $T_{h}{ }^{i}$, 当冷流体流过热电单元 $i$, 一部分热量 $q_{L}{ }^{i}$ 由热电模块冷端表面释放到冷流 体, 随之冷流体温度由 $T_{c}{ }^{i+1}$ 升为 $T_{c}{ }^{i}$, 模块冷表面 温度降至 $T_{L}{ }^{i}$, 其他剩余的能量 $P_{t e g}$ 则转换为热电 功率输出。对于一个计算单元 $i$, 电流 $(I)$ 和热平衡方 程如下

$$
\left\{\begin{array}{l}
I=\left[\sum_{i=1}^{n_{x}} n_{y} \alpha_{p n}\left(T_{h}^{i}-T_{L}^{i}\right)\right] /\left(2 n_{x} n_{y} R_{p n}\right) \\
q_{h}^{i}=0.5 c_{f} m_{f}\left(T_{f}^{i}-T_{f}^{i+1}\right)=n_{y} L w k_{f}\left[0.5\left(T_{f}^{i}+T_{f}^{i+1}\right)-T_{h}^{i}\right] \\
q_{h}^{i}=L w k_{f}\left[0.5\left(T_{f}^{i}+T_{f}^{i+1}\right)-T_{h}^{i}\right]=\alpha_{p n} I T_{h}^{i}+K_{p n}\left(T_{h}^{i}-T_{L}^{i}\right)-0.5 I^{2} R_{p n} \\
q_{L}^{i}=L w k_{c}\left[T_{h}^{i}-0.5\left(T_{c}^{i}+T_{c}^{i+1}\right)\right]=\alpha_{p n} I T_{L}^{i}+K_{p n}\left(T_{h}^{i}-T_{L}^{i}\right)+0.5 I^{2} R_{p n} \\
q_{L}^{i}=0.5 c_{c} m_{c}\left(T_{c}^{i+1}-T_{c}^{i}\right)=n_{y} L w k_{c}\left[T_{h}^{i}-0.5\left(T_{c}^{i}+T_{c}^{i+1}\right)\right]
\end{array}\right.
$$

式中, $c_{f}$ 和 $c_{c}$ 分别为热流体和冷流体的比热容, $m_{f}$ 和 $m_{c}$ 分别是热流体和冷流体流量, $L$ 是换热器总长 度, $w$ 是换热器总宽度; $\alpha_{p n}$ 表示赛贝克系数, $K_{p n}$ 表示导热率, $R_{p n}$ 表示一对 $\mathrm{PN}$ 结的电阻, $k_{f}$ 表示
热流体至热电模块热表面间的总传热系数, $k_{c}$ 表示 热电模块冷表面至冷流体间的总传热系数, 热电性 能相关参数的计算方程如下

$$
\left\{\begin{array}{l}
\alpha_{p n}=\alpha_{p}-\alpha_{N} \\
K_{p n}=c_{1} c_{2}\left(\lambda_{p}+\lambda_{N}\right) / c_{3} \\
R_{p n}=c_{3}\left(\rho_{p}+\rho_{N}\right) /\left(c_{1} c_{2}\right) \\
k_{f}=\left(R_{f}+R_{h 1}+R_{h 2}+R_{h 3}+R_{f c o n}\right)^{-1}=\left(1 / h_{f}+\delta_{e x c} / \lambda_{e x c}+\delta_{c o p} / \lambda_{c o p}+\delta_{c e r} / \lambda_{c e r}+R_{f c o n}\right)^{-1} \\
k_{c}=\left(R_{c}+R_{L 1}+R_{L 2}+R_{L 3}+R_{c c o n}\right)^{-1}=\left(1 / h_{c}+\delta_{e x c} / \lambda_{e x c}+\delta_{c o p} / \lambda_{c o p}+\delta_{c e r} / \lambda_{c e r}+R_{c c o n}\right)^{-1}
\end{array}\right.
$$

式中, $\alpha_{p} 、 \rho_{p} 、 \lambda_{p} 、 \alpha_{N} 、 \rho_{N}$ 和 $\lambda_{N}$ 分别是 $\mathrm{P}(\mathrm{N})$ 半导体 臂的赛贝克系数、电阻率、热导率; $c_{1} 、 c_{2} 、 c_{3}$ 分 别是 $\mathrm{P}(\mathrm{N})$ 半导体臂的长度、宽度和高度; $\delta_{e x c} 、 \delta_{c o p}$ 、 $\delta_{c e r}$ 分别是换热器板、铜片和陶瓷片的厚度; $\lambda_{\text {exc }}$ 、 $\lambda_{c o p}$ 和 $\lambda_{\text {cer }}$ 分别是换热器板、铜片和陶瓷片的导热系 数, $h_{f}$ 和 $h_{c}$ 分别为热流体和冷流体的对流换热系数。

外部负载电阻与热电模块内电阻取相等, 计算 如下

$$
R_{L}=n_{x} n_{y} R_{p n}
$$

总热电功率输出为

$$
P_{\text {teg }}=2 \sum_{i=1}^{n_{x}}\left(q_{h}^{i}-q_{L}^{i}\right)
$$
功 ${ }^{[18]}$

废气流过通道时由于流动阻力所需要消耗的原

$$
P_{\text {pump }}=f_{z}\left(m_{f} / \rho_{f}\right)
$$

式中, $f_{z}$ 是尾气流过热端换热器的压降, $\rho_{f}$ 是废气流 体的密度。

因此, 热电系统净输出功率为

$$
P_{n e t}=P_{t e g}-P_{\text {pump }}
$$

\section{2 锯齿错列肋片尾气换热器模型}

采用锯齿错列肋片如图 2 所示, 它是指不同肋 片区域相互错开一定间隔而形成的间断式肋片，从 而增加换热流体的湍流度。此外, 由于各肋片间距 长度较短、不连续, 热边界层在其上还来不及增长 就断裂, 从而使得它具有传热系数高、结构紧凑等 优点, 被广泛应用于各种强化换热的场合。其中 $s, h$, $l$ 和 $t$ 分别为锯齿错列肋片的胁片宽度, 肋片高度、 肋片长度 (本文取 $l=5 \mathrm{~mm}$ ) 和胁片厚度 (本文取 $t=0.2 \mathrm{~mm}$ ), $H$ 为换热器通道高度(本文取 $H=5 \mathrm{~mm}$ )。 此外, 当后文分析中肋宽或肋高不作为变化量时, 肋宽取 $s=5 \mathrm{~mm}$, 肋高取 $h=4.8 \mathrm{~mm}$ 。 


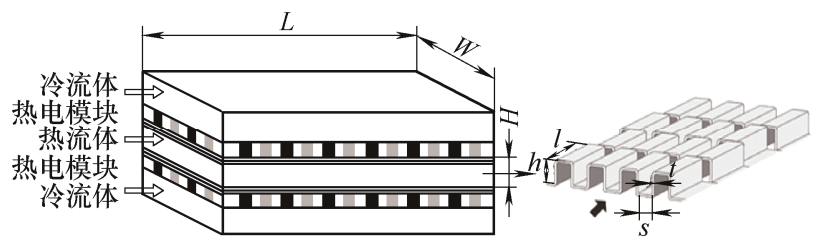

图 2 锯齿错列肋片尾气换热器结构

汽车尾气流过锯齿错列肋片热端换热器进行换 热后产生的压降为 ${ }^{[18]}$

$$
f z=4 f\left(L / D_{f}\right)\left(\rho_{f} u_{f}^{2} / 2\right)
$$

式中, $\rho_{f}$ 和 $u_{f}$ 分别为气体密度和流速, $D_{f}$ 为当量 直径, $f$ 为范宁摩擦因子, 可由以下关联式计算 ${ }^{[20]}$

$$
D_{f}=4 s h l /[2(s l+h l+t h)+t s]
$$

$f=9.6243 \operatorname{Re}_{f}^{-0.7422}(s / h)^{-0.1856}(t / l)^{0.3053}(t / s)^{-0.2659} \times$ $\left[1+7.669 \times 10^{-8} \operatorname{Re}_{f}^{4.429}(s / h)^{0.92}(t / l)^{3.767}(t / s)^{0.236}\right]^{0.1}$

汽车尾气流过锯齿错列肋片的 Colburn 因子 $(j)$ 可由以下关联式计算 ${ }^{[19]}$

$$
\begin{aligned}
& j=0.6522 \operatorname{Re}_{f}^{-0.5403}(s / h)^{-0.1541}(t / l)^{0.1499}(t / s)^{-0.0678} \times \\
& {\left[1+5.269 \times 10^{-5} \operatorname{Re}_{f}^{1.34}(s / h)^{0.504}(t / l)^{0.456}(t / s)^{-1.055}\right]^{0.1}}
\end{aligned}
$$

因此, 对流换热系数 ${ }^{[19]}$

$$
h_{f}=N u_{f} \lambda_{f} / D_{f}=j \operatorname{Re}_{f} \operatorname{Pr}_{f}^{1 / 3}
$$

式中, $\lambda_{f}$ 是废气热导率。

为了从流场和温度场相互配合的角度揭示对流 传热物理机制, 本文引用对流传热场协同理论, 流 场与热流场的协同性越好, 对流强化传热的效果也 越好。流场与热流场的协同程度可以用场协同数表 征 ${ }^{[20]}$

$$
F_{c}=N u_{f} /\left(\operatorname{Re}_{f} P r_{f}\right)
$$

\section{2 计算方法}

电流 $(I)$ 由热电模块内部温度分布所决定, 然而 热电温度分布又受电流影响, 因此热电系统电流与 内部温度分布相互耦合, 这里由 Fortran 程序迭代 实现。同样, 传热系数 $\left(k_{f}\right)$ 和内部温度分布相互耦合,

\begin{tabular}{|c|c|c|c|}
\hline 物理量 & 数值 & 物理量 & 数值 \\
\hline 半导体臂长度 $c_{1} / \mathrm{m}$ & 0.005 & 热流体流量 $m_{f} /(\mathrm{g} / \mathrm{s})$ & 46 \\
\hline 半导体臂宽度 $c_{2} / \mathrm{m}$ & 0.005 & 冷却水流量 $m_{c} /(\mathrm{g} / \mathrm{s})$ & 500 \\
\hline 半导体臂高度 $c_{3} / \mathrm{m}$ & 0.005 & 热流体进口温度 $T_{\text {fin }} /{ }^{\circ} \mathrm{C}$ & 550 \\
\hline $\begin{array}{l}\text { 冷却水对流换热系 } \\
\text { 数 } h_{c} /\left(\mathrm{W} \cdot \mathrm{m}^{-2} \cdot \mathrm{K}^{-1}\right)\end{array}$ & 1000 & $\begin{array}{c}\text { 冷流体进口温度 } \\
T_{c i n} /{ }^{\circ} \mathrm{C}\end{array}$ & 80 \\
\hline $\begin{array}{c}\mathrm{P} \text { 半导体塞贝克系数 } \\
\alpha_{p} / \times 10^{-4}(\mathrm{~V} / \mathrm{K})\end{array}$ & 2.037 & $\begin{array}{c}\mathrm{N} \text { 半导体塞贝克系数 } \\
\alpha_{N} / \times 10^{-4}(\mathrm{~V} / \mathrm{K})\end{array}$ & -1.721 \\
\hline $\begin{array}{l}\mathrm{P} \text { 半导体电阻率 } \\
\rho_{p} / \times 10^{-5}(\Omega \cdot \mathrm{m})\end{array}$ & 1.314 & $\begin{array}{l}\mathrm{N} \text { 半导体电阻率 } \\
\rho_{N} / \times 10^{-5}(\Omega \cdot \mathrm{m})\end{array}$ & 1.119 \\
\hline $\begin{array}{c}\mathrm{P} \text { 半导体热导率 } \\
\lambda_{p} /\left(\mathrm{W} \cdot \mathrm{m}^{-1} \cdot \mathrm{K}^{-1}\right)\end{array}$ & 1.265 & $\begin{array}{c}\mathrm{N} \text { 半导体热导率 } \\
\lambda_{N} /\left(\mathrm{W} \cdot \mathrm{m}^{-1} \cdot \mathrm{K}^{-1}\right)\end{array}$ & 1.011 \\
\hline
\end{tabular}
同样由程序迭代实现。热流体流经温差发电器时温 度在不断降低, 其物性参数也在不断变化, 这里采 用空气物性作为热流体物性参数。热电模块采用 $\mathrm{Bi}-\mathrm{Te}$ 型热电材料, 热电系统基本计算参数见表 1 。
表 1 热电系统基本计算参数

为了验证本文所建数学模型计算结果的可靠 性, 模拟计算结果与试验对比验证结果如图 3 所示。 图 3 中，将采用本程序所得的计算结果与文献[21] 的试验数据进行了对比。试验中热电模块热端设置 加热面积为 $40 \mathrm{~mm}^{2}$ 的加热器, 试验热流量 $Q_{h}$ 分别 采用 $10 \mathrm{~W} 、 20 \mathrm{~W} 、 30 \mathrm{~W} 、 40 \mathrm{~W}$ 和 $50 \mathrm{~W}$, 试验中 选用的热电模块类型为 TEG1-127-1.4-1.6, 结构尺 寸为 $40 \mathrm{~mm} \times 40 \mathrm{~mm} \times 3.8 \mathrm{~mm}$, 冷却方式为水冷却。 模拟计算中采用与文献试验相同的热电工况及物性 参数, 分别计算了不同热流量下的热电输出功率情 况。图中圆点为采用自建模型所获得的计算数据,

图中曲线则为文献试验数据, 对比可以看出, 模拟 计算结果与试验结果有很好的吻合，误差在 $2 \%$ 以 内, 由此验证了本文所建模型及自编计算程序的正 确性。

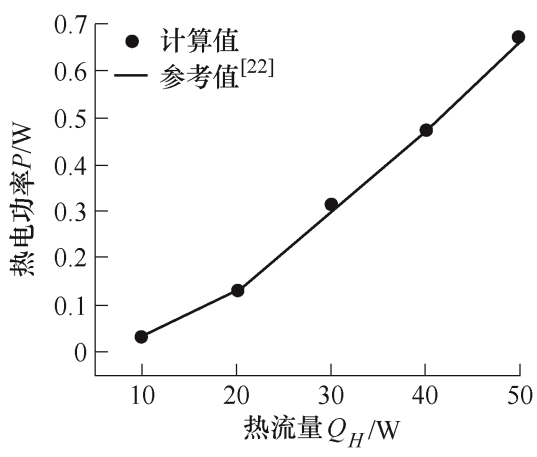

图 3 模拟结果与试验结果对比

\section{3 计算结果分析}

\section{1 肋高和胁宽对热电功率影响及场协同分析}

对于具有固定热电模块尺寸(这里长和宽分别 取 $0.3 \mathrm{~m}$ 和 $1.2 \mathrm{~m}$ )的锯齿错列肋片尾气换热器, 内部 结构尺寸对热电性能会产生一定的影响。这里将重点 基于场协同强化换热机理，分析胁片宽度和肋片高度 对热电器总功率、泵功, 以及净功率输出的影响。 
图 4 为不同胁宽下的热电功率输出及场协同 数。由图可以看出, 随着肋宽的增加, 热电器的总 输出功率在不断降低, 当肋宽大于 $6 \mathrm{~mm}$ 时, 其变 化趋势不再明显。分析其内在原因, 可由场协同数 的变化进行解释: 随着肋宽增加, 场协同数不断降 低, 直至肋宽大于 $6 \mathrm{~mm}$ 时变化不明显; 场协同数 的降低意味着换热器内部对流换热效果的减弱, 对 流换热减弱时必然导致热电系统总输出功的减少。 但由图也可以看出, 小的肋宽时场协同效果虽好, 但强化换热效果增强的同时也产生了较大的流阻, 导致相应较高的洜功消耗。综合来看, 由于肋宽变 化引起的总输出功和洜功同向变化, 且变化幅度很 相近, 由此导致系统净输出功反而受肋宽的影响很 小, 基本保持不变。



图 4 不同肋宽下的热电功率输出及场协同数

图 5 为不同肋高下的热电功率输出及场协同 数。由图可以看出, 随着肋高的增加, 热电器的总 输出功率不断降低, 当肋高大于 $4 \mathrm{~mm}$ 时, 其变化 趋势变得不明显。进一步关注场协同数的变化, 类 同图 4 的分析可以解释上述变化。但小的肋高时场 协同效果虽好，强化换热效果增强的同时仍带来了 较大的流阻, 对应具有较高的潅功消耗。由图可以 看到, 相比之对总功率的影响幅度, 肋高对葲功率 的影响幅度更大些。因此, 综合作用结果导致系统 净输出功随肋高的增加而增加, 当肋高大于 $4 \mathrm{~mm}$ 时增加变得不再明显。



图 5 不同肋高下的热电功率输出及场协同数

\section{2 不同肋宽、肋高下的热电模块总尺寸优化}

根据前期发表的基于光滑平板式换热器的研究 成果 ${ }^{[22]}$, 对于选定的尾气换热器型式, 当考虑流阻 时, 存在最优热电模块尺寸使得净输出功最大。这 里进一步研究采用强化换热时, 肋片宽度和肋片高 度对热电模块最优尺寸以及相应最优热电性能的影 响。分析计算时，对于不同的肋片结构尺寸，取热 电模块的长和宽为优化变量, 取热电器最大净输出 功为优化目标, 取得峰值功率时获得的各变量均称 为相应的最优变量, 计算结果见图 6 11。

图 6 为不同肋宽下最大净功率输出以及对应的 最优对流换热系数。由图可以看出, 较小的肋宽可 以获得较大的净输出功, 且对应具有较大的最优对 流换热系数。

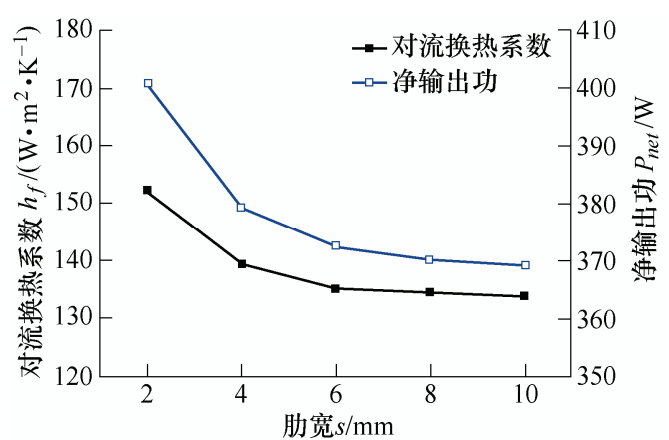

图 6 不同肋宽下最大净输出功及最优对流换热系数

图 7 为不同肋宽下获得最大净功率输出时的最 优热电模块长度和宽度尺寸。由图可知, 当肋片宽 度不同时, 热电器获得最大净输出功时所需要的最 优热电模块尺寸差异也较大。热电模块的最优长度 尺寸随肋宽增加而明显增加，而最优宽度尺寸则随 肋宽增加而明显降低。因此, 针对不同肋片结构参 数进行热电模块的最优尺寸设计是十分必要的。

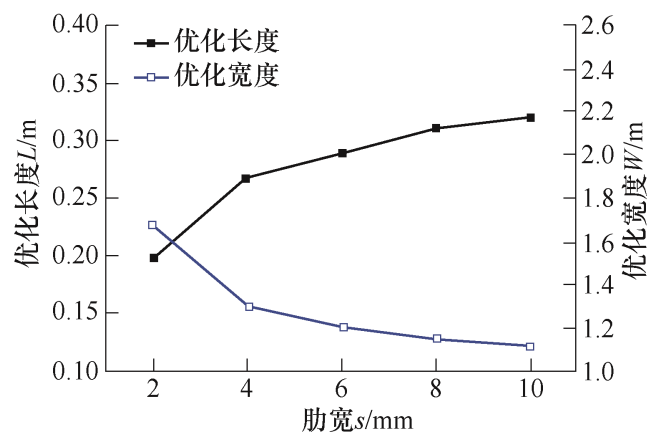

图 7 不同肋宽下获得最大净输出功时的最优热电模块尺寸

图 8 为不同肋宽下获得最大净功率输出时的最 优热电模块面积 $(A)$ 以及单位面积净输出功率 $\left(P_{p e r}\right)$ 。这 里, $A=L \times w, P_{p e r}=P_{n e t} A$ 。由图 8 可见, 肋宽由 $2 \mathrm{~mm}$ 增加至 $4 \mathrm{~mm}$ 时, 最优热电模块总面积也随之增加, 
但当肋宽大于 $4 \mathrm{~mm}$ 后, 最优总面积则受肋宽影响 很小。总体来看, 尽管热电模块的最优长度和最优 宽度尺寸受胁宽的影响较大, 但总的最优热电模块 面积变化并不明显。但单位面积最大净输出功却受 肋宽的影响较大, 随肋宽的增加而明显降低。

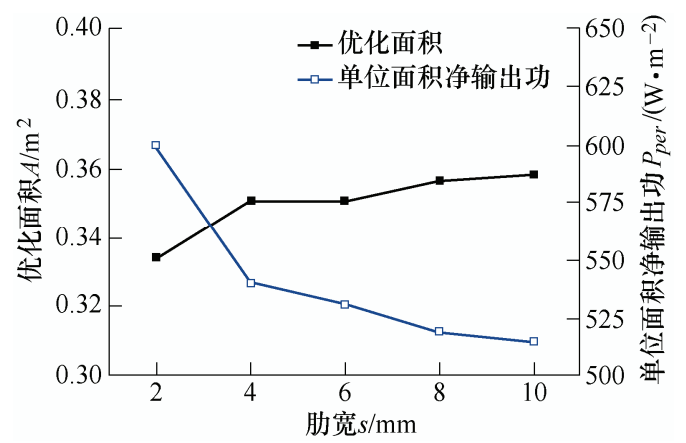

图 8 不同肋宽下最优热电模块面积及单位面积净输出功

图 9 为不同肋高下最大净输出功输出以及对应 的最优对流换热系数。由图可以看出, 较大的肋高 可以获得较大的净输出功, 但增幅不明显; 相比于 肋宽, 肋高对最优对流换热系数的影响也不大。



图 9 不同肋高下最大净输出功率及最优对流换热系数

图 10 为不同肋高下获得最大净功率输出时的 最优热电模块长度尺寸和宽度尺寸。可以看出, 热 电模块的优化长度随肋高增加而明显增加, 而模块 的最优宽度尺寸则随肋高增加而明显降低, 这与胁 宽的影响规律相同。而且, 无论对于肋宽还是肋高 结构, 设计为小的肋片尺寸时, 最优热电模块结构 为短宽型, 设计为大的肋片尺寸时, 最优热电模块 结构为窄长型。由于热电模块尺寸与尾气换热器结 构相对应匹配, 因此, 肋片结构参数也将对尾气换 热器结构尺寸优化设计有重要的影响。

图 11 为不同胁高下总的最优热电模块面积及 单位面积净输出功率。由图 11 可知, 肋高对最优热 电模块总面积的影响较小, 肋高从 $1 \mathrm{~mm}$ 左右增加 至 $2 \mathrm{~mm}$ 左右时, 单位面积最大净输出功有一定的 增加, 但肋高再增加时, 单位面积最大净输出功基 本保持不变。总体上看, 肋高变化对热电器优化性
能的影响并不明显。

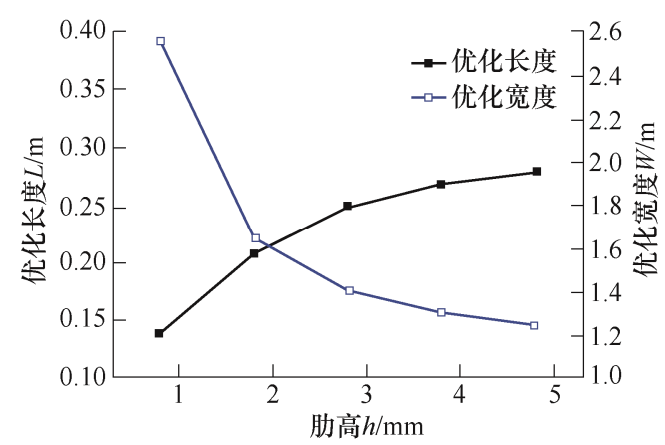

图 10 不同肋高下获得最大净功率输出时最优热电模块尺寸

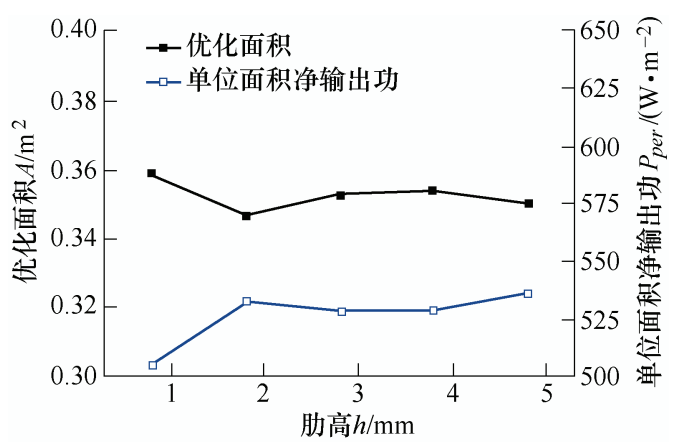

图 11 不同肋高下最优热电模块面积及单位面积净输出功

\section{4 结论}

针对锯齿错列肋片尾气换热器型式, 研究不同 肋片结构对热电系统优化性能的影响, 获得的主要 结论如下。

（1）对于固定尺寸热电模块, 通过强化对流换 热的场协同理论分析，肋片宽度和高度越小，场协 同效果越好, 热电器总输出功也越高; 但进一步考 虑流阻作用下的泵功消耗, 肋宽对热电器净功率输 出影响较小, 但净输出功随肋高的增加而增加。

(2) 存在最优的热电模块尺寸使得温差发电器 取得最大的净功率输出, 热电模块的最优长度和宽 度尺寸都随肋宽和肋高的变化而明显变化, 肋宽和 肋高为小尺寸时, 最优热电模块结构为短宽型, 反 之为窄长型。

(3) 较小的肋宽可以获得较大的净输出功, 单 位面积最大净输出功随胁宽的增加而明显降低; 较 大的肋高可以获得较大的净输出功, 但增幅不明显, 单位面积最大净输出功基本不受肋高的影响。

\section{参 考 文 献}

[1] 任大海, 卢凯, 戴震宇, 等. 基于微机电系统技术的微 型热电致冷器研究进展 [J]. 机械工程学报, 2010, 
46(8): 114-120.

REN Dahai, LU Kai, DAI Zhenyu, et al. Research progress of micro thermoelectric cooler based on MEMS technology[J]. Journal of Mechanical Engineering, 2010, 46(8): 114-120.

[2] 李运泽, 魏传锋, 袁领双, 等. 应用热电制冷器的微型 航天器主动温度控制及仿真 $[\mathrm{J}]$. 机械工程学报, 2005, 41(10): 149-152.

LI Yunze, WEI Chuanfeng, YUAN Lingshuang, et al. Active temperature control and simulation of micro spacecraft using thermoelectric refrigerator[J]. Journal of Mechanical Engineering, 2005，41(10): 149-152.

[3] 陈林根, 孟凡凯, 戈延林, 等. 半导体热电装置的 热力学研究进展 $[\mathrm{J}]$. 机械工程学报, 2013, 49(24): 144-154.

CHEN Lingen, MENG Fankai, GE Yanlin, et al. Progress in thermodynamics of semiconductor thermoelectric devices[J]. Journal of Mechanical Engineering, 2013, 49(24): 144-154.

[4] 赵付舟, 汪秀敏, 梁荣, 等. 汽车热电废热回收的现状 及关键技术分析 $[\mathrm{J}]$. 小型内燃机与摩托车, 2013 , 42(2): 93-96.

ZHAO Fuzhou, WANG Xiumin, LIANG Rong, et al. Current status and key technical analysis of automotive thermal power waste heat recovery[J]. Small Internal Combustion Engines and Motorcycles, 2013, 42(2): 93-96.

[5] DENG Zongquan, CUI Jinsheng, HOU Xuyan, et al. Calibration of discrete element heat transfer parameters by central composite design[J]. Chinese Journal of Mechanical Engineering, 2017, 30(2): 1-9.

[6] 李小龙, 谢长君, 黄亮, 等. 基于一种新混合法的车载 尾气热电发电最大功率跟踪研究 $[\mathrm{J}]$. 机械工程学报, 2019, 55(16): 132-140.

LI Xiaolong, XIE Changjun, HUANG Liang, et al. Maximum power tracking of vehicle exhaust gas thermoelectric generation based on a new hybrid method[J]. Journal of Mechanical Engineering, 2019, 55(16): 132-140.

[7] 陈威, 李筠, 杨海马, 等. 基于 ANSYS 的温差发电器 耦合设计分析[J]. 电子测量技术, 2018, 41(24): 18-22. CHEN Wei, LI Jun, YANG Haima, et al. Coupling design analysis of thermoelectric generator based on ANSYS[J]. Electronic Measurement Technology, 2018, 41(24): $18-22$
[8] YANG Lixin, ZHOU Mengjun, TIAN Zihao. Heat transfer enhancement with mixing vane spacers using the field synergy principle[J]. Chinese Journal of Mechanical Engineering, 2017, 30(1): 127-134.

[9] FRÉDÉRIC J L, ÉRIC V S, LALANDE-BERTRNAD N. A study on heat transfer enhancement using flow channel inserts for thermoelectric power generation[J]. Energy Conversion and Management, 2013， 75(5): 532-541.

[10] LU Hongliang, WU Ting, BAI Shengqinag, et al. Experiment on thermal uniformity and pressure drop of exhaust heat exchanger for automotive thermoelectric generator[J]. Energy, 2014, 54(54): 372-377.

[11] NIU Zhiqiang, DIAO Hai, YU Shuhai, et al. Investigation and design optimization of exhaust-based thermoelectric generator system for internal combustion engine $[\mathrm{J}]$. Energy Conversion and Management, 2014, 85 : 85-101.

[12] BAI Shengqiang, LU Hongliang, WU Ting, et al. Numerical and experimental analysis for exhaust heat exchangers in automobile thermoelectric generators $[\mathrm{J}]$. Case Studies in Thermal Engineering, 2014(4): 99-112.

[13] SHI Yongming, CHEN Xin, DENG Yuan, et al. Design and performance of compact thermoelectric generators based on the extended three-dimensional thermal contact interface $[\mathrm{J}]$. Energy Conversion and Management, 2015(106): 110-117.

[14] ESARTE J, MIN G, ROWE D M. Modelling heat exchangers for thermoelectric generators $[\mathrm{J}]$. Journal of Power Sources, 2001, 93(1): 72-76.

[15] BAKER C, VUPPULURI P, SHI L, et al. Model of heat exchangers for waste heat recovery from diesel engine exhaust for thermoelectric power generation[J]. Journal of Electronic Materials, 2012, 41(6): 1290-1297.

[16] LU Chi, WANG Shixue, CHEN Chen, et al. Effects of heat enhancement for exhaust heat exchanger on the performance of thermoelectric generator[J]. Applied Thermal Engineering, 2015(89): 270-279.

[17] WANG Tongcai, LUAN Weiling, LIU Tongjun, et al. Performance enhancement of thermoelectric waste heat recovery system by using metal foam inserts[J]. Energy Conversion and Management, 2016(124): 13-19.

[18] MAVRIDOU S, MAVROPOULOUS G C, BOURIS D, et al. Comparative design study of a diesel exhaust gas heat exchanger for truck applications with converntinal 
and stage of the art heat transfer enhancements[J]. Appl. Therm. Eng., 2010, 30: 935-947.

[19] MANGLIK R M, BERGLES A E. Heat transfer and pressure drop correlations for the rectangular offset strip fin compact heat exchanger[J]. Experimental Thermal and Fluid, 1995, $10(2)$ : 171-180.

[20] 李志信, 过增元. 对流传热优化的场协同理论[M]. 北 京: 科学出版社, 2010 .

LI Zhixin, GUO Zengyuan. Field synergy theory for convective heat transfer optimization [M]. Beijing : Science Press, 2010.
[21] ZHOU Zeguang, ZHU Dingsheng, WU Hongxia, et al. Modeling, experimental study on the heat transfer characteristics of thermoelectric generator[J]. Journal of Thermal, 2013(1): 48-54.

[22] HE Wei, WANG Shixue, ZHANG Xing, et al. Optimization design method of thermoelectric generator based on exhaust gas parameters for recovery of engine waste heat[J]. Energy, 2015, 91(11): 1-9.

作者简介: 何为(通信作者), 女, 1982 年出生, 讲师。主要研究方向为 余热能半导体温差发电技术以及数据中心冷却技术等。

E-mail: weihe@tjcu.edu.cn 